\title{
Head and Neck Squamous Cell Carcinoma - Comparative Evaluation of Pathological Parameters in Young and Old Patients
}

\author{
Samreen $\mathrm{Naz}^{1}$, Kanwal Salah${ }^{2}$, Amna Khurshid ${ }^{1 *}$, Atif Ali Hashmi ${ }^{1}$, Naveen \\ Faridi $^{1}$
}

\begin{abstract}
Background: To evaluate pathological features of head and neck squamous cell carcinoma (HNSCC) and to compare these pathological features in patients younger and older than $\mathbf{4 0}$ years. Materials and Methods: All resection specimens of HNSCC between 2010 and 2013 evaluated. Tumor characteristics - grade, location (site) cervical node status, alongwith presence or absence of extranodal extension, lymphovascular invasion, gender and age - were extracted from surgical pathology reports. Results: Among these $n=19$ patients $(\mathbf{2 1 . 8 \% )}$ were 40 years or younger and $n=68$ patients $(78.2 \%)$ were above 40 years of age. The mean age was $34(20-40$ years) in the younger group and $56(42-86)$ in the older group. The most common location of HNSCC in both groups was the oral cavity. The analysis of histopathological features including grade of tumor, tumor size, extranodal extension and comparison between two groups do not show any significant difference. Conclusions: There are no specific pathological characteristics of HNSCC in young patients. An interesting observation is that exposure to expected risk factors is similar in both groups, in younger patients they have less time to act and yet tumors are the same in terms of tumor size, lymph node status and lymphovascular invasion. Therefore further research is recommended to look for potentiating factors.
\end{abstract}

Keywords: Squamous cell carcinoma - head and neck - young and old patients - Pakistan

Asian Pac J Cancer Prev, 16 (9), 4061-4063

\section{Introduction}

More than 500,000 people worldwide are diagnosed with squamous cell carcinoma (SCC) of Head and Neck each year (Saba et al., 2011). Head and neck cancer is the fifth most common cancer worldwide and is the most common neoplasm in central Asia (Parkin et al., 1999). Also keeping in mind the statistics published by Karachi cancer Registry (KCR) that Head and Neck cancers are accounted for approximately one fifth $(21 \%)$ of the cancers in males and one tenth (11\%) in females ( Bhurgri et al., 2006). The present study is conducted with the objective of reviewing clinicopathological parameters and association with age. This is because an increasing number of young patients are being affected worldwide, with up to $5.5 \%<$ 40 years (Toner et al., 2009) .

Head and neck SCC is an important health issue. The WHO predicts a continuing world wide increase in the number of patients with oral cancer, extending this trend into next several decades (Scuibba et al., 2001).

Head and neck cancers, 10.0-10 (international classification of Diseases 10th revision) include cancers of the lip, oral cavity, pharynx and larynx, head and neck cancers are grouped together with the justification of similar natural history, risk factors, morphology and control measures. The head and neck cancers mainly comprise are self inflicted and preventable as indulgence in risk factors is avoidable.

But in some parts of world, Indian sub-continent oral cancer is a major problem. The two year data of Karachi cancer registry, showed that it is the second most common malignancy in males and females (Bhurgri et al., 1998). Indians like Pakistanis in the same sub-continent share preventable risk factors like regular pan eating and smooking. Head and neck cancers amongst the commonest malignancies, according for approximately $20 \%$ of cancer burden in India. (Siddiqui et al., 2013)

One objective of conducting this study is review of clinicopathological features of Head and neck SCC. Another important objective is to analyze/discuss controversy in the up coming literature regarding the differences in clinicopathological features of Head and neck SCC in young patients. Head and Neck SCC in the young: a spectrum or distinct group.

The purpose of this study is to evaluate the pathological features of head and neck SCC and to compare these clinicopathological features upto 40 years and more than 40 years. 


\section{Materials and Methods}

This descriptive study is conducted at histopathology department of Liaquat National Hospital. All resection specimen of Head and Neck SCC diagnosed between 2010 and 2013 included in the study excluding salivary gland lesion. Patients with other malignancies like thyroid tumors lymphomas and Metastatic tumor were excluded. In this study, a young patient is defined and being 40 or younger. Pathological parameters including tumor size and lymph nodes were sub categorized according to AJCC 2011. The other characteristic evaluated include grade, location, sex and age. The analysis was done using SPSS version. The variable were analyzed using chi-square, $\mathrm{P}$ value $<0.05$ was considered statically significant.

\section{Results}

The pathology reports of 87 patients of Head and neck squamous cell carcinoma diagnosed between 2010 and 2013 at Liaquat national Hospital were evaluated. All these cases were resection specimens of which 19 cases were less than and equal to 40 and 68 were more than 40 years. The mean age in whole group is 51 years. The mean age was 34 (age range 20-40) in young group and 56 years, (age range 42-86) in older group.

The male female ratio is same in both old and young group. Most lesions in young and old group involved oral cavity i.e., $63 \%$ and $58 \%$ respectively. The only difference

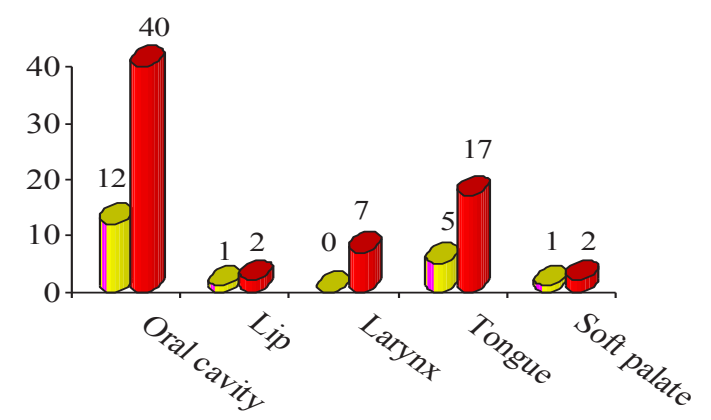

Figure 1. Age and Site Distribution of Head and Neck Squamous Cell Carcinoma

Table 1. Distribution of Pathological Parameters of Head and Neck Squamous Cell Carcinoma

\begin{tabular}{lccc}
\hline Tumor Invasion & $\leq 40$ Yrs & $>40$ Yrs & p. Value \\
\hline T1 & $6(6.9 \%)$ & $15(21 \%)$ & 0.494 \\
T2 & $10(11.5 \%)$ & $33(37.9 \%)$ & \\
T3 & $3(3.4 \%)$ & $20(23.0 \%)$ & \\
Lymph Node Status & $\leq 40$ Yrs & $>40$ Yrs & p. Value \\
N0 & $6(31.6 \%)$ & $31(35.5 \%)$ & 0.168 \\
N1 & $8(42.1 \%)$ & $11(16.2 \%)$ & \\
N2a & $0 \%$ & $1(1.5 \%)$ & \\
N2b & $5(26.2 \%)$ & $20(29.4 \%)$ & \\
N2c & $0 \%$ & $1(1.1 \%)$ & \\
Histologic Grade & $\leq 40$ Yrs & $>40$ Yrs & p. Value \\
G I & $4(22.2 \%)$ & $20(29.4 \%)$ & 0.298 \\
G II & $13(72.2 \%)$ & $36(52.9 \%)$ & \\
G III & $1(5.6 \%)$ & $12(17.6 \%)$ & \\
Lymphovascular Invasion & $\leq 40$ Yrs & $>40 \mathrm{Yrs}$ & p. Value \\
Not present & $18(94.7 \%)$ & $68(100 \%)$ & 0.218 \\
Present & $1(5.3 \%)$ & $0 \%$ & \\
\hline
\end{tabular}

in the location of tumor is that larynx is the third common site (10\%) in older group but none of the case of laryngeal carcinoma was diagnosed in young group. (Figure 1) A comparison and distribution of the pathological parameters including tumor size, lymph node status, histological grade and lymphovascular invasion between young and older group is show in table 1 . There is no statistically significant difference in these pathological parameters

\section{Discussion}

Head and neck SCC usually affect middle aged or elderly men and has strong association with smoking and alcohol consumption (Blot et al., 2002).

Although recent studies have shown increased incidence of head and neck SCC among young adults (Schantz et al., 2002).

In this study we retrospectively reviewed and compared the pathology of lesions from young patients with carcinoma of $\mathrm{H}$ and $\mathrm{N}$ with lesions from older patients in order to determine if there are any defining characteristics in the young population.

Head and neck SCC is known to affect more males than females. Most studies have reported similar higher number of males (Schantz et al., 2002).

However few studies show decrease of male to female ratio in sex distribution of young patients and patel etal reported that SCC of the oral cavity, specially tongue, is increasing among young white individuals and that the increasing trend was significantly greater in young white women compared with young white man (Schantz et al., 2002; Toner and O Regan 2009).

This change in the incidence can be due to increased trends of smoking or drinking in women and the greater prevalence in men can be caused by their more exposure to occupational carcinogens, toxins and other risk factors like manjuana compared to women. In our study only 21 of the patients were females, possibly reflecting the existing difference in prevalence of smoking or drinking between Pakistani men and women. It appears that geographic locations and social life styles among different world populations might have accounted for these variations. This is the same observation as in a study conducted by Oral- Maxilofacial Department of China an obvious male predominance coincided with smoking trend among Chinese young individuals and female patients were more likely to have no traditional risk factors such as smoking or drinking.(Fan et al., 2014).

In our study, the majority of our patients suffered from oral cavity SCC $(n=52)$ and tongue SCC $(n=22)$. This finding is compatible with other Asian studies. As in our study Asian population usually suffer from cancer of the buccal mucosa due to betel quid / tabaco chewing habits ; Buccal mucosa SCC constitutes the largest group. This observation is seen in both young and old age group. In literature in majority of studies tongue is reported as most common location for Head and Neck SCC in young adults. Our finding are also compatible with data of Karachi cancer registry (Patel et al., 2011) .

It has been reported that in Karachi mucosa cheek was the most common site for oral cancer $(55.9 \%)$ followed 
by tongue, palate, gum and lip. This further emphasize the point that the difference in subsite of involvement in two geographical areas is probably indicative of difference in risk factors involved in two continents.

Oral cavity and Oropharyngeal SCC in the young patients has been linked with history of exposure to Human papilloma virus, Herpes simplex virus (HSV), human immunodeficiency virus (HIV) etc. The fact that even when these young patients indulge in known risk factors of alcohol and smoking, it is for considerably shorter periods compared to adult counterparts as to induce oral carcinogenesis leaves room for further search as to other responsible etiological factors. We cannot however draw any conclusions from our study as regard risk factorstumor location association since our retrospective review did not include evaluation of any of viral risk factors. HPV has association with development of head and neck squamous cell carcinoma as reported in few publishing Asian studies. (Rushatamukayanunt et al., 2014) However other studies do not document HPV as etiological agent of head and squamous cell carcinoma particularly in young patients. (Gan et al., 2014). We recommend study for evaluation of HPV in young Pakistani patients.

In our study, majority of patients were diagnosed as T2 and majority of the patients show positive nodal status. Head and neck cancer if detected in early stage are highly curable but unfortunately as shown by our data and also same depicted by Karachi cancer registry (Bhurgri et al., 2006; Patel et al., 2011). This is also true for our neighbour India where tumor also present at advanced stage (Krishnamurthy et al., 2013). This observation is different from a study conducted at Japan showing statistically significant difference between tumor stage between young and older patients. The greater number of older patient presented with large lesions (TU). The diagnosis relies on patient presentation and pick up by physician. This is high time that physicians, Gps and patients should be sensitized about diagnosis. So the precious time between first presentation to clinician and final confirmation can be shortened. Indicates the necessity of training for physicians, GPs, other health workers at community levels. This reflects the delays of early diagnosis. Like Iranian study lymph nodes which did not different significantly in our younger population (Sasaki et al., 2005). Regard to pathological features including tumor grade, lymph node status, tumor size, lymphovascular invasion, depth of tumor we did not find any statistically significant difference between young and older age groups-so this confirms previous studies which are either unable to detect or there is little evidence that lesions of head and neck in younger individuals are histologically different from older individual (Mafi et al., 2012).

As we already know about the well established strong influence of clinical stage on prognosis emphasis the importance of early diagnosis and treatment of Head \& Neck Squamous Cell Carcinoma but similar pathological finding in both groups does not suggest that younger patients should be managed differently.

In conclusion, the study shows that there are no specific pathological characteristics of Head and Neck carcinomas in young adults. Although by observation it appears that exposure to behavioral risk factors like eating Pan and smoking is shared by young and older groups. In younger individuals they have less time to act and yet lesions are similar in terms of histological pathological parameters. Further research is needed to investigate possible confounding or potentiating factors including genetic, hereditary, diet and demographic factors..

\section{References}

American Joint Committee on Cancer. AJCC Cancer staging manual 7th ed. Published by Springer Verlage New York, NY.

Bhurgri Y, Rahim A, Bhutto K, et al (1998). Karachi cancer Registry. Pages with refrence to book, from 321-325.

Bhurgri Y, Bhurgri A, Usman A, et al (2006). Epidemiological review of head and neck cancers in Karachi. Asian Pac J Cancer Prev, 2, 195-200.

Blot WJ, McLaughlin JK, Winn DM, et al (1998). Smoking and drinking in relation to oral and pharyngeal cancer. Cancer Res, 48, 3282-7

Fan Y, Zheng L, Mao MH, et al (2014). Survival analysis of oral squamous cell carcinoma in a subgroup of young patients. Asian Pac J Cancer Prev, 15, 8887-91.

Gan LL, Zhang H, Guo JH, Fan MW (2014). Prevalence of human papillomavirus infection in oral squamous cell carcinoma: a case-control study in Wuhan, China. Asian Pac J Cancer Prev, 15, 5861-5.

Krishnamurthy A, Ramshankar V (2013). Early stage oral tongue cancer among non-tobacco users an increasing trend observed in a South Indian patient population presenting at a single centre. Asian Pac J Cancer Prev, 4, 5061-65.

Mafi N, Kadivar M, Hosseini N, Ahmadi S, Zare-Mirzaie (2012). Head and neck squamous cell carcinoma in Iranian patients and risk factors in young adults: a fifteen-year study. Asian Pac J Cancer Prev, 13, 3373-8.

Parkin DM, Pisani P, Ferlay J. 1999. Global cancer Statistics. CA Cancer J Clin, 1, 33-64.

Patel SC, Carpenter WR, Tyree S, et al (2011). Increasing incidence of oral tongue squamous cell carcinoma in young white women, age 18 to 44 years. J Clin Oncol, 11, 1488-94.

Rushatamukayanunt P, Morita K, Matsukawa S, et al (2014). Lack of association between high- risk human papillomaviruses and oral squamous cell carcinoma in young Japanese patients. Asian Pac J Cancer Prev, 15, 4135-41.

Saba NF, Goodman M, Ward K, et al (2011). Genderand ethentic disperities in incidence and survival of squamous cellcarcinomaof the oral tongue, base of the tongue and tonsils:a survelience epidemiology and end results programbased analysis. Oncology, 1, 12-20.

Sasaki T, Moles DR, Imai Y, Speight PM (2005).Clinicopathological features of squamous cell carcinoma of the oral cavity in patients $<40$ years of age. J Oral Pathol Med, 3, 129-33

Schantz SP, Yu GP (2002). Head and Neck 1973-1997 with special analysis for tongue cancer. Arch Otolaryngol Head and Neck Surg, 3, 268-74.

Sciubba JJ (2001). Oral cancer. The importance of early diagnosis and treatment. AM J Clin Dermatol, 4, 239-51.

Siddiqui MS, Chandra R, Aziz A, Suman S. (2012). Epidemiology and histopathological spectrum of head and neck cancers in Bihar, a state of Eastern India. Asian Pac J Cancer Prev, 13, 3949-53.

Toner M, O'Regan EM (2009). Head and neck squamous cell carcinoma in the young: a spectrum or a distinct group? Part1. Head Neck Pathol, 3, 246-8. 